\title{
Strontium Migration at the GDC-YSZ Interface of Solid Oxide Cells in SOFC and SOEC Modes
}

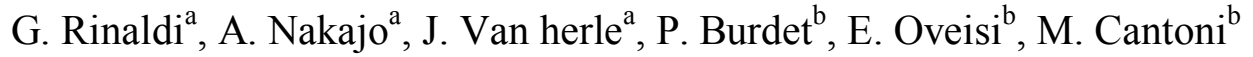 \\ ${ }^{\text {a }}$ Group of Energy Materials (GEM), Faculty of Engineering Sciences and Technology \\ STI, Ecole Polytechnique Fédérale de Lausanne, Lausanne, Switzerland \\ ${ }^{\mathrm{b}}$ Interdisciplinary Centre for Electron Microscopy (CIME) \\ Ecole Polytechnique Fédérale de Lausanne, Lausanne, Switzerland
}

\begin{abstract}
Strontium migration from the oxygen electrode to the interface between the electrolyte and interlayer was investigated in solid oxide cells in fuel cell (SOFC) and electrolysis (SOEC) modes. Four samples were imaged by focused ion beam scanning electron microscopy (FIB-SEM) serial sectioning. After reconstruction, the spatial distribution of the strontium zirconate secondary phase was examined by measuring the volume fractions, phase size distributions, interfacial surface areas and the shape of each detected distinct inclusion. The analysis shows that the accumulation lingers during operation. The results also suggest that the inclusions tend to propagate towards the GDC/YSZ interface. The detrimental effect on the performance was assessed using 3-D finite element transport analysis, by comparing the effective conductivity computed with and without the presence of the secondary phase. The operation in SOFC mode caused a limited decrease of the conductivity, while the effects on the performance are more significant for SOEC operation.
\end{abstract}

\section{Introduction}

Solid Oxide Fuel Cells (SOFC) and Electrolysis Cells (SOEC) represent a promising energy conversion technology because of their high conversion efficiency and operational flexibility. The degradation rate of the state-of-the art is however just above the threshold for widespread market entry. In order to increase the long-term stability and consequently the economic viability, the degradation rates need to be reduced (well) below $1 \% \mathrm{kh}^{-1}$ under practical conditions. One contribution to the degradation arises from the chemical interaction between the oxygen electrode (typically lanthanum-cobalt-strontium oxides with perovskite structure) and its environment (1). Sr evaporates in the form of hydroxide and/or migrates by surface, solid or gas-phase diffusion (2). In the case of yttriastabilized-zirconia (YSZ) electrolytes, Zr can also diffuse, and react with Sr forming a secondary $\mathrm{SrZrO}_{3}$ phase at the interface, along with closed porosities (3).

Zirconate formation is known to occur during the fabrication sintering of the cell layers (above $1000^{\circ} \mathrm{C}$ ) with a critical effect on the initial cell performance. Hence, a gadolinium-doped-ceria (GDC) layer is inserted between the electrolyte and oxygen active electrode to prevent the formation of this secondary phase without compromising the transport of oxygen ions. In commercial cells, the GDC layer is often screen-printed 
for costs reasons. Full densification is difficult to achieve with this deposition method, resulting in a partially porous microstructure. Therefore, only part of the thickness of the blocking layer efficiently limits cation diffusion and $\mathrm{SrZrO}_{3}$ can be found also in pristine cells (4). Furthermore, several studies reported enhanced accumulation upon operation in SOEC mode $(5,6)$. The mechanism remains however partially understood and the knowledge on the evolution during operation and the effect on the performance is limited. $\mathrm{SrZrO}_{3}$ inclusions partially obstruct the oxygen ion conduction paths and force transport through smaller sections, increasing the effective ohmic resistance and local ohmic heating. They are located within an interdiffusion region between the YSZ and ceriabased layer, which forms during the sintering process already. The solid-state reaction results in the formation of a thin and convoluted interaction zone, which is most extended in the electrolyte and exhibits an ionic conductivity lower by two orders of magnitudes compared to YSZ (3). Zr migration also results in the formation of closed porosities along the YSZ grain boundaries leading to a mechanical weakening of the interface (7). The presence of voids combined with thermal cycles may provoke delamination of the interface.

The purpose of this study is a quantitative 3-D characterization of the evolution of the zirconate secondary phase during operation in both SOFC and SOEC modes and of the effect on the effective conductivity of the interface.

\section{Experimental}

The pristine and cells aged under SOFC conditions were composed by a nickel-yttria stabilized zirconia (Ni-YSZ) support electrode on the fuel feed side, a YSZ electrolyte, a GDC compatibility layer and a lanthanum strontium cobalt ferrite (LSCF)-based oxygen electrode. The SOEC aged sample comprised instead a LSC-based oxygen electrode. Table 1 lists the footprint area and GDC length for the four volumes examined. The results of the analysis were normalized by the footprint area because of the different reconstructed volumes. The effective thickness of the GDC layer was estimated as the distance in the $\mathrm{Z}$ direction between the position of the first $\mathrm{SrZrO}_{3}$ when scanning from the YSZ side and the location where the volume fractions of pores and GDC are equal (Figures 1a-1b). The testing conditions of the four analyzed samples are summarized hereafter:

- V1: pristine cell, after Ni reduction.

- V2 (SOFC-mode): segmented cell exposed for $1900 \mathrm{~h}$ to air and dry $\mathrm{H}_{2}$ diluted with $\mathrm{N}_{2}$ and $750^{\circ} \mathrm{C}$, maintained most of the time at open-circuit voltage, except for discrete EIS measurement sequences.

- V3 (SOFC-mode): 6-cell short stack operated for $4700 \mathrm{~h}$ with dry $\mathrm{H}_{2}$ at $0.4 \mathrm{~A} \mathrm{~cm}^{-}$ 2 and $780^{\circ} \mathrm{C}$ for most of the time.

- V4 (SOEC-mode): 6-cell short stack tested for $10700 \mathrm{~h}$ at $0.6 \mathrm{~A} \mathrm{~cm}^{-2}$ for the first $3250 \mathrm{~h}$ and at $0.5 \mathrm{~A} \mathrm{~cm}^{-2}$ afterwards, at $720^{\circ} \mathrm{C}$ (feed flow composition of $90 \%$ $\mathrm{H}_{2} \mathrm{O}$ and $10 \% \mathrm{H}_{2}$ ). Detailed information on the testing conditions of this stack is provided in (8). 
TABLE I. Dimensions of the reconstructed volumes.

\begin{tabular}{lcc}
\hline Volume sample & $\begin{array}{c}\text { Footprint } \\
\text { area }\left(\boldsymbol{\mu ~ m}^{2}\right)\end{array}$ & $\begin{array}{c}\text { Estimated GDC layer } \\
\text { thickness }(\boldsymbol{\mu m})\end{array}$ \\
\hline V1: pristine & 71.54 & 5.7 \\
V2: SOFC, $1900 \mathrm{~h}$ & 41.71 & 3.8 \\
V3: SOFC, $4700 \mathrm{~h}$ & 45.16 & 3.8 \\
V4: SOEC, $10700 \mathrm{~h}$ & 106.58 & 3.1 \\
\hline
\end{tabular}

a.

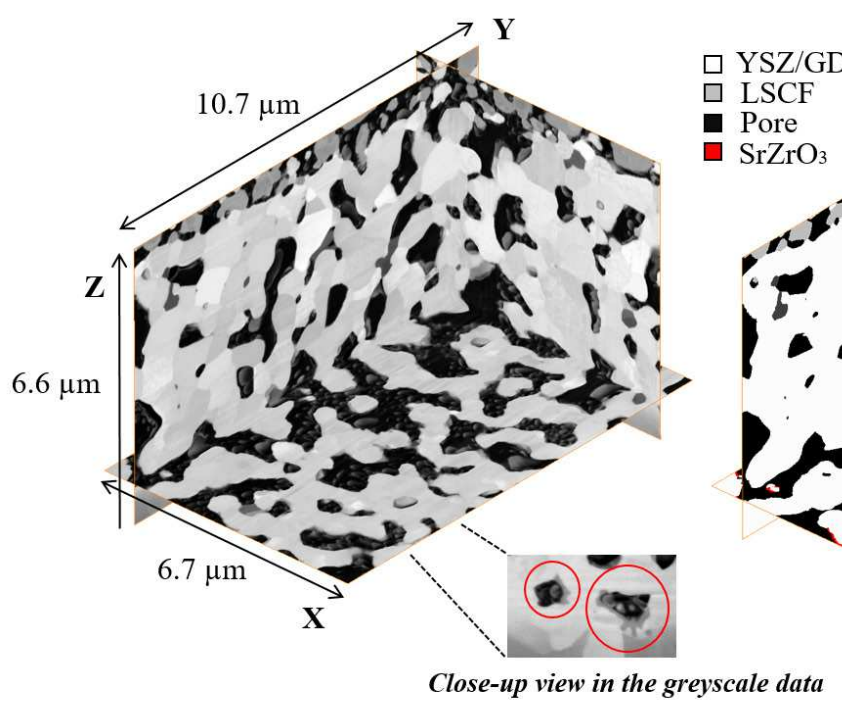

b.

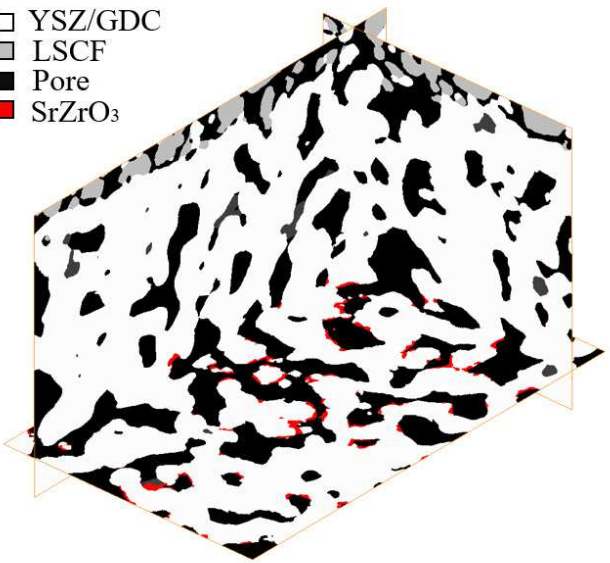

Figure 1. 3-D view of the GDC interlayer region. (a): ortho-slices in the EsB data of the V1 (pristine) reconstruction with a close-up view of the detected features with a distinct grayscale value and (b) same volume after segmentation.

The selected samples were cut and embedded in resin to fill the pores in the microstructure. Focused ion beam-scanning electron microscopy (FIB-SEM) serial sectioning was performed with an acceleration voltage of 1.7 or $1.8 \mathrm{kV}$ and dual channel acquisition (secondary electron/secondary ion (SESI) or inLens, and energy selective backscattered (EsB) detectors). 3-D energy-dispersive X-ray spectroscopy (EDX) elemental mapping was performed for the sample V4 only, by changing the acceleration voltage automatically every 10 sections to $10 \mathrm{kV}$, which allowed the detection of $\mathrm{La}, \mathrm{Sr}$, $\mathrm{Co}, \mathrm{O}, \mathrm{Gd}, \mathrm{Ce}, \mathrm{Zr}$ and Y (Figure 2a). The 3-D EDX maps were resampled at the electron images sizes and registered. The alignment and thickness of the slices was controlled using fiducial marks milled before the acquisition to guarantee isometric voxels, with a precision in the range of $1 \mathrm{~nm}$. The isometric voxel size was 6.3-10 nm, depending upon the sample.

3-D EDX enabled to confirm that features with a distinct grayscale and located at the YSZ/GDC interface contain $\mathrm{Sr}$ and $\mathrm{Zr}$. The measurements did not reveal a clear continuous Sr-gradient across the GDC compatibility layer (Figure 2a). Previous post-test analysis of the SOEC V4 sample by transmission electron microscopy (TEM)-EDX with selected area electron diffraction (SAED) confirmed the presence of a $\mathrm{SrZrO}_{3}$ secondary phase (8) at the interface between the YSZ electrolyte and GDC layer, which corresponds to the grayscale features in the FIB-SEM dataset. In the V4 case, delamination just below the YSZ/GDC interface was observed over large areas at several locations, in both 2-D and 3-D analyses (see Figures 2a-2b). 
a.

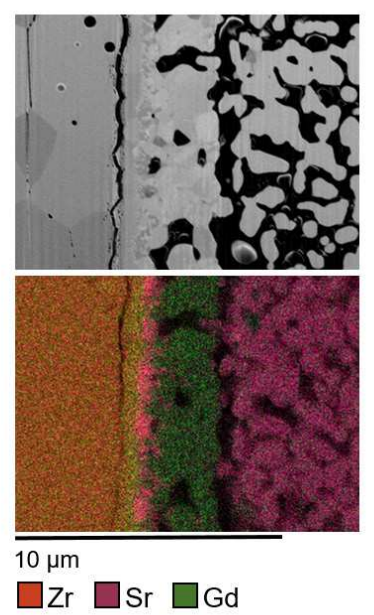

b.

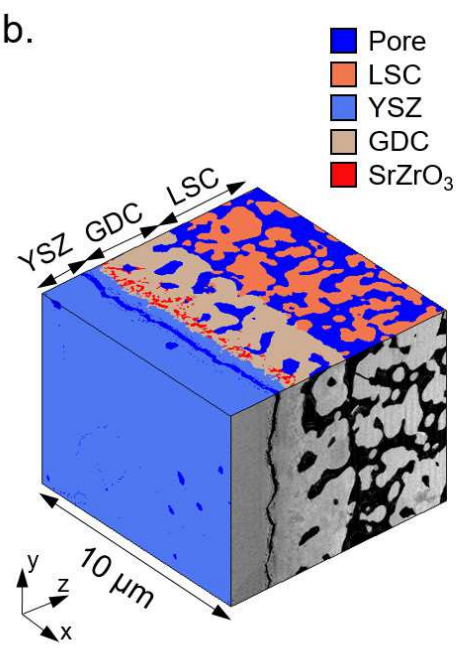

Figure 2. (a) Electron image and corresponding EDX map from a section in the 3-D serial sectioning dataset of the sample aged in SOEC mode for $10700 \mathrm{~h}$, including the YSZ electrolyte, the GDC compatibility layer and oxygen electrode. (b) Reconstruction of the full dataset (grayscale electron images and segmented volume).

The segmentation, which consists in assigning to each voxel belonging to a same material phase the same label, was based on the grayscale electron images using Matlab routines with calls to Avizo for image gradient and watershed transform computations. Further descriptions of the methods used for image processing and quantitative 3-D analyses are provided in (9). Figure $1 \mathrm{a}$ and $2 \mathrm{~b}$ show overall and close-up 3-D views of the aligned grayscale and segmented datasets. The $\mathrm{Sr}$ inclusions can be identified because of their darker level of grey (Figures $1 a-2 b$ ). For the present study where 3-D EDX elemental mapping was not performed systematically, YSZ and GDC phases were treated as a single phase, because of their similar grayscale levels.

\section{Analysis}

A series of metric and topological properties of the detected strontium zirconate phase was measured to identify the mechanisms of formation and to comprehend whether or not a detrimental evolution occurs in the long-term. The evolution after SOFC and SOEC operation was examined by measuring the volume fractions, interfacial surface areas, 3-D phase size distribution and connectivity. For the purpose of this analysis, the region of interest is limited to the GDC layer and its interface with the YSZ electrolyte.

\section{$\underline{\text { Volume Fraction }}$}

Figure 3 displays scans of the volume fraction of the $\mathrm{SrZrO}_{3}, \mathrm{YSZ} / \mathrm{GDC}$ and pore phases, measured slice by slice along the perpendicular Z-direction. The Sr-inclusions were detected already in the pristine sample (V1), with a maximum in the slice volume fraction of $3.5 \%$. The scans clearly indicate that the inclusions are located at the beginning of the GDC layer, at the start of the pores phase. The GDC layer is thicker in the pristine sample. This does however not seem to impede the diffusion process more efficiently, since the sample after $1900 \mathrm{~h}$ of SOFC exposure (V2) exhibits a similar volume fraction profile, despite a thinner GDC layer. The amount of secondary phase is 
higher after $4700 \mathrm{~h}$ in SOFC short stack operation (V3) and is the highest in the sample operated in SOEC mode for $10700 \mathrm{~h}$, with a peak in the slice volume fraction reaching $25 \%$, and a wider spread across the interface with respect to the other cases (approximately $1.6 \mu \mathrm{m}$ instead of $0.8 \mu \mathrm{m}$ ). As a general trend, the accumulation seems to scale with test duration under polarization.

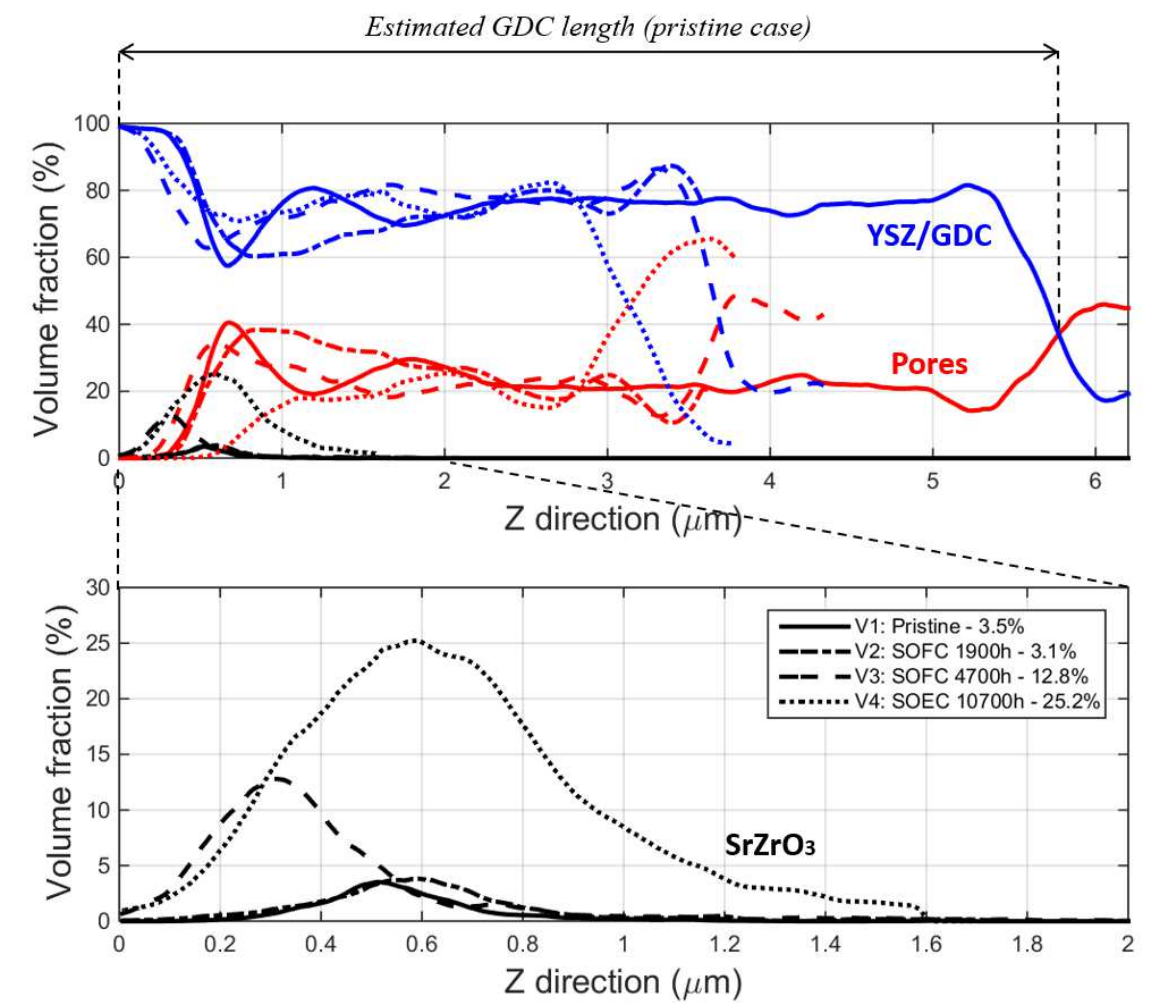

Figure 3. Volume fraction scans along the $\mathrm{Z}$ direction, perpendicular to the interface. The peak values of the $\mathrm{SrZrO}_{3}$ volume fraction are listed in the legend.

\section{$\underline{\text { Interfacial Surface Areas }}$}

The interfacial surface areas (ISA, Table II) provide further information about the morphology and location of the inclusions within the YSZ/GDC interface. The second column lists the volume of detected $\mathrm{SrZrO}_{3}$ per interface footprint area. The data confirms that the secondary phase quantity increases during operation. In the third and fourth column are the ISA between Sr and pores and YSZ/GDC, respectively. The subset of the interfacial surface area in contact with GDC/YSZ appears to increase with time, contrarily to that with pores. The trend is clearly observed in the ratio (column 5 in Table II). This indicates that the $\mathrm{Sr}$ zirconate phase tends to become further included in the microstructure during operation, up to being mostly embedded in the V4 case.

TABLE II. Specific volume per interface footprint area and interfacial surface to volume ratios of the $\mathrm{SrZrO}_{3}$ secondary phase.

\begin{tabular}{ccccc}
\hline Volume & $\begin{array}{c}\text { Specific Vol } \\
\mathbf{S r Z r O}_{\mathbf{3}} \\
\left(\boldsymbol{\mu m}^{\mathbf{3}} / \boldsymbol{\mu m}^{\mathbf{2}}\right)\end{array}$ & $\begin{array}{c}\text { ISA Sr-Pore } \\
\left(\boldsymbol{\mu m}^{2} / \boldsymbol{\mu m}^{\mathbf{3}}\right)\end{array}$ & $\begin{array}{c}\mathbf{I S A}_{\text {Sr-GDC }} \\
\left(\boldsymbol{\mu m}^{2} / \boldsymbol{\mu m}^{\mathbf{3}}\right)\end{array}$ & $\begin{array}{c}\text { Ratio Sr-GDC/ } \\
\text { Sr-Pore }(-)\end{array}$ \\
\hline V1 & 0.011 & 26.9 & 47.4 & 1.8 \\
V2 & 0.016 & 15.6 & 41.1 & 2.6 \\
V3 & 0.048 & 15.7 & 42.2 & 2.7 \\
V4 & 0.168 & 1.6 & 52.0 & 32.0 \\
\hline
\end{tabular}




\section{$\underline{\text { Phase Size Distribution }}$}

The phase size distribution measurements shown in Figure 4 indicate that the morphology of the secondary phase differs in the samples aged in SOFC and SOEC, as anticipated by the decrease in total interfacial surface area (Table II). The pristine case is characterized by a smaller size with a peak at $0.1 \mu \mathrm{m}$. The size of the inclusions is larger in the samples aged in either SOFC or SOEC mode (see decrease in the $0.1 \mu \mathrm{m}$ peak, and increase in the 0.3-0.5 $\mu \mathrm{m}$ range of the distributions in Figure 4). While the two SOFC cases have similar size distributions despite the different operational time, the SOEC volume (V4) presents a further accretion. The volume fraction, ISA and phase size distribution measurements suggest that the secondary phase is initially dispersed, small and in contact with the pore phase. The decrease of the total surface to volume ratio is linked to an increase of the size distribution and a decrease of the share in contact with the pore phase, suggesting a shift towards a dendritic-like pattern. After $10700 \mathrm{~h}$ of SOEC operation, the amount of secondary phase is significant enough to result in a change in morphology.

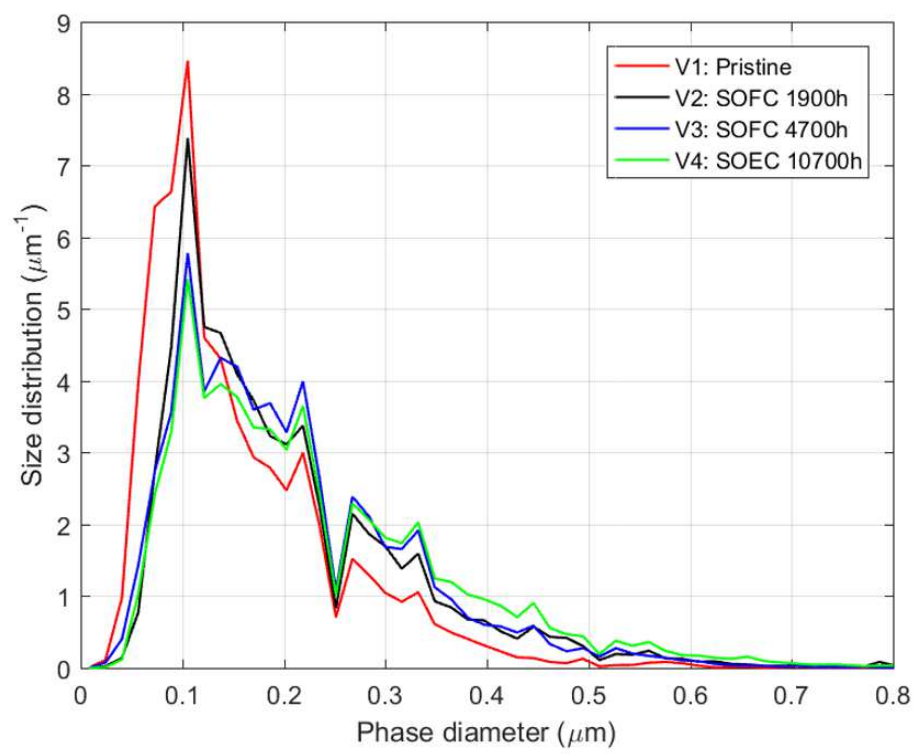

Figure 4. Phase size distribution of the $\mathrm{SrZrO}_{3}$ secondary phase in the pristine sample and after ageing in SOFC and SOEC mode.

\section{Characterization of Individual Sr Zirconate Inclusions}

The analysis of the individual $\mathrm{SrZrO}_{3}$ regions detected in each sample was conducted to study further differences in morphology. The segmented $\mathrm{SrZrO}_{3}$ regions were first labelled, then each individual region characterized. The regions smaller than 8 voxels were not included in the analysis (Table III).

TABLE III. Number-based analysis of detected $\mathrm{SrZrO}_{3}$ regions.

\begin{tabular}{|c|c|c|c|c|}
\hline Volume & $\begin{array}{c}\text { Number of } \\
\mathrm{SrZrO}_{3} \text { regions } \\
\text { per } \\
\text { footprint area }\end{array}$ & $\begin{array}{c}\text { Fraction } \\
\text { considered } \\
\text { for analysis }\end{array}$ & $\begin{array}{c}\text { Percentage totally } \\
\text { enclosed in GDC }(\%)- \\
\text { corresponding fraction of } \\
\mathrm{SrZrO}_{3} \text { volume (\%) }\end{array}$ & $\begin{array}{c}\text { Average } \\
\text { inclusion } \\
\text { volume } \\
\left(\mu \mathrm{m}^{3}\right)\end{array}$ \\
\hline V1 & 1.6 & 0.97 & $0.9-0.3$ & 0.0071 \\
\hline V2 & 1.5 & 1.00 & $12.5-1.1$ & 0.0107 \\
\hline V3 & 5.4 & 0.45 & $16.3-0.1$ & 0.0197 \\
\hline V4 & 14.5 & 0.99 & $68.8-2.7$ & 0.0117 \\
\hline
\end{tabular}


The density function of the region size resembles that of a Pareto distribution, the majority with relatively small dimension and, on the other hand, a few much larger. As shown in Table III, all the individual regions in the pristine sample (V1) are in contact with the pore phase. After SOFC and SOEC aging, about $12-16 \%$ and $69 \%$ of the inclusions are embedded in the GDC phase, respectively, but they represent a small portion of the total volume. After operation in SOEC mode (V4), the $\mathrm{SrZrO}_{3}$ secondary phase is present as many small and more convoluted regions (which lower the number weighted average volume compared to the V3 case), but most of the volume forms largely extended and connected regions.

Figure 5a displays the volume-weighted sphericity $(\Sigma)$ distribution of the inclusions. This parameter informs on how close the shape of a particle approaches that of a perfect sphere $(\Sigma \leq 1)$. The shape deviates gradually from a sphericity of $0.15-0.35$ (V1, pristine) down to 0.02-0.05 (V4, SOEC). Figure $5 \mathrm{~b}$ provides the distribution of the ratio of the interfacial surface area of $\mathrm{SrZrO}_{3}$ between GDC and pores, similarly to the fifth column of Table II, but here for each inclusion. These two plots suggest that the regions grow towards the YSZ electrolyte depending on the operation time, with a severe alteration

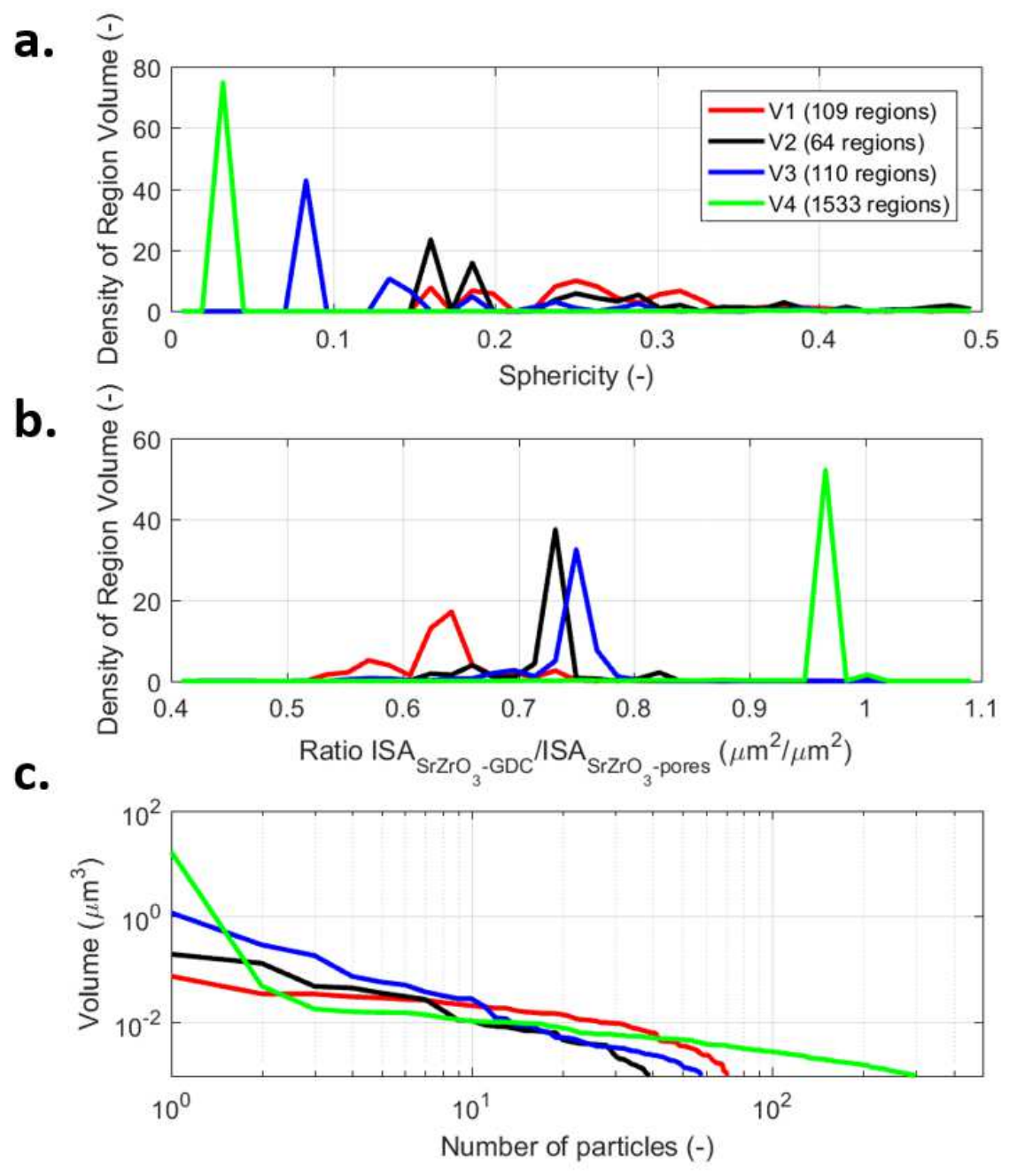

Figure 5. Volume weighted sphericity (a) and surface area to volume ratio (b) distribution of the detected individual $\mathrm{SrZrO}_{3}$ inclusions. (c) Volumes of the individual inclusions sorted from the largest $(\mathrm{x}=1)$ to the smallest. 
after SOEC aging. Figure 5c shows the volume of each particle sorted from the largest $(\mathrm{x}=1)$ to the smallest and displayed in logarithmic scale. The most extended regions are therefore reported on the leftward part of the plot, while increasing $\mathrm{x}$ values refer to ever smaller individual inclusions. V4 SOEC volume contains both the largest and the smallest inclusion, even if their number is one order of magnitude higher than in the other volumes. Figure $5 \mathrm{c}$ indicates that the size of the largest particle increases monotonically with operation duration.

\section{Transport Analysis}

The conductivity of $\mathrm{SrZrO}_{3}$ is four orders of magnitude lower than that of GDC (respectively $8.5 \times 10^{-7} \mathrm{~S} / \mathrm{cm}$ at $800^{\circ} \mathrm{C}$ and $0.087 \mathrm{~S} / \mathrm{cm}$ at $750^{\circ} \mathrm{C}$ ) $(3,10)$. Finite-element (FE) transport simulations were performed to quantify the effect of the detected inclusions alone on the effective ionic conductivity of the YSZ electrolyte/GDC compatibility layers together. The performance degradation was assessed by comparing two cases. The transport analysis was first performed by implementing the ratio of the ionic conductivities mentioned above. Then, an ideal limiting case was simulated by treating the Sr-inclusions as GDC/YSZ, i.e. with the same conductivity. The ratio of the two cases was then computed to obtain a global increase in resistivity. This simplified analysis does not distinguish the difference in the detrimental effect on transport of the subset of the secondary phase enclosed in the GDC and that located mostly on the external surface.

The simulations were performed in Abaqus with periodic boundary conditions imposed across the Z-direction and no-flux boundary conditions on the other $\mathrm{x}$ and $\mathrm{y}$ directions. The four volumes considered were aligned on the peak of the $\mathrm{Sr}$ volume fraction and cropped at $2.1 \mu \mathrm{m}$ further along the Z-direction. The footprint areas are those listed in Table I. An additional GDC/YSZ layer of $0.9 \mu \mathrm{m}$ (reaching a total length of 3.0 $\mu \mathrm{m}$ ) was added for imposing the periodic boundary conditions on corresponding pairs of points on opposing surfaces (11). The computational domains were generated by converting the voxels into $1^{\text {st }}$ order brick elements. The volumes were resampled by a factor of 4 in each direction to keep the RAM memory requirements below $256 \mathrm{~GB}$ in all cases. Simulations on test volumes with a size of $3.5 \mu \mathrm{m}^{3}$ showed that the resampling results in an increase of the effective conductivity of $0.9 \%$.

The effect of the $\mathrm{SrZrO}_{3}$ on the simulated distribution of current is shown in Figures 6a-6b. The presence of extended regions may further hinder the ionic current, leading to a significant local increase of flux. Whether or not the additional ohmic losses can cause noticeable local increases of the temperature with detrimental consequences on the mechanical stability of the interface remains an unclarified question. 


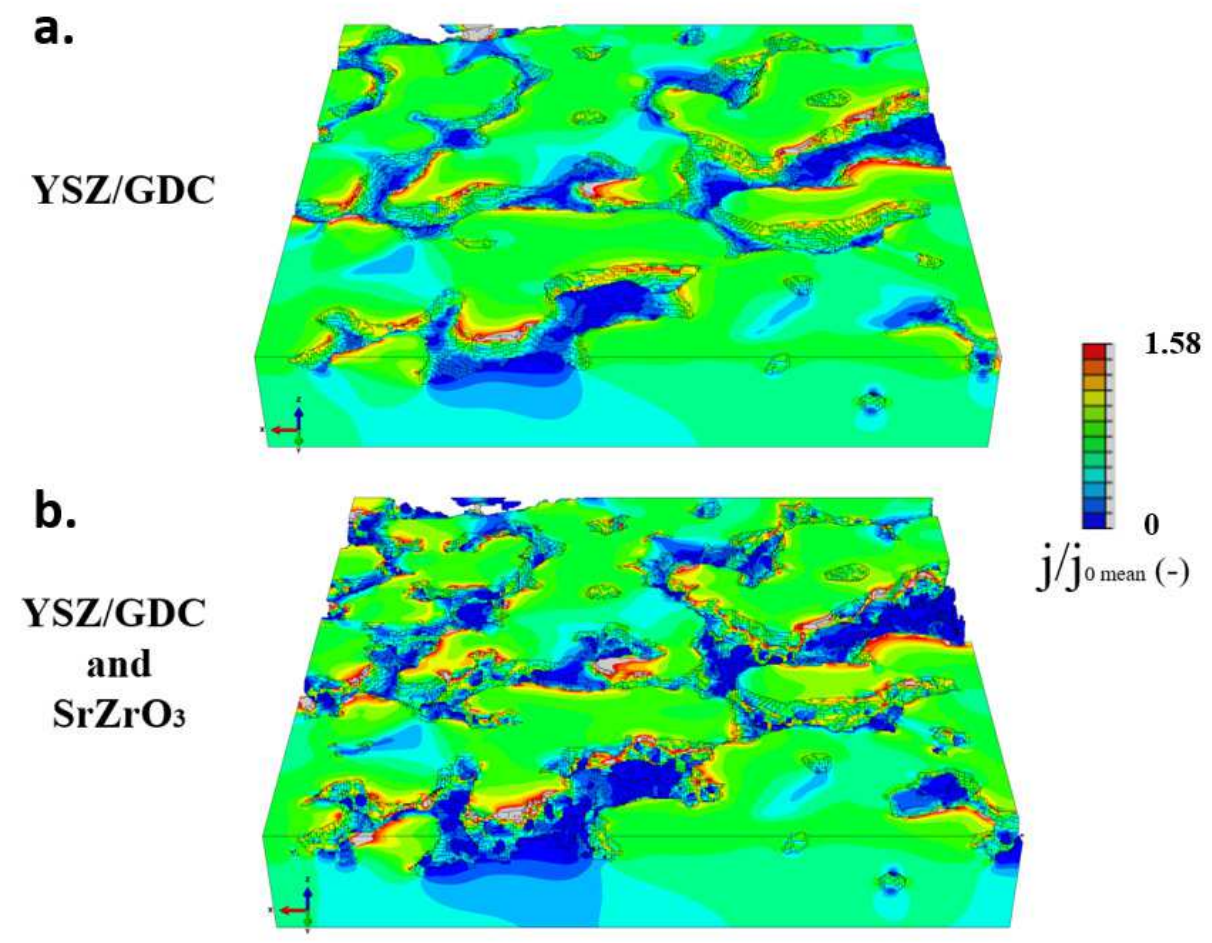

Figure 6. Simulated distribution of the ionic current in the V3 sample (SOFC, $4700 \mathrm{~h}$ ). (a) $\mathrm{SrZrO}_{3}$ treated as part of the GDC/YSZ ion-conducting phase, (b) as close to insulating, as in the real microstructure.

The increase in resistivity computed for each volume is provided in Table IV. The detrimental effect of the zirconate phase is the highest for the V4 volume (SOEC), leading to an increase in the effective resistivity of $13 \%$. Figure 7 suggests at first appraisal a close to linear dependence on the operation time, which is however most likely misleading. The estimates of the rate of increase in resistivity and specific Srvolume are $0.6 \% \mathrm{kh}^{-1}$ and $1.1 \% \mathrm{kh}^{-1}$ in SOFC and SOEC mode, respectively. The inclusions detected in the pristine volume $\mathrm{V} 1$ and formed during the sintering process have a limited effect on the performance, estimated to $1.5 \%$. After $1900 \mathrm{~h}$ at OCV (V2), the increase in resistivity is similar to the pristine case (V1). The degradation of the effective conductivity is more significant after $4700 \mathrm{~h}$ in SOFC mode (V3). In general, there is a clear correlation between the surface specific volume of $\mathrm{SrZrO}_{3}$ and the decrease in effective conductivity.

TABLE IV. Results of the transport analysis

\begin{tabular}{cc}
\hline Volume & Increase in resistivity (\%) \\
\hline V1 & $1.49 \%$ \\
V2 & $1.50 \%$ \\
V3 & $4.13 \%$ \\
V4 & $12.91 \%$ \\
\hline
\end{tabular}




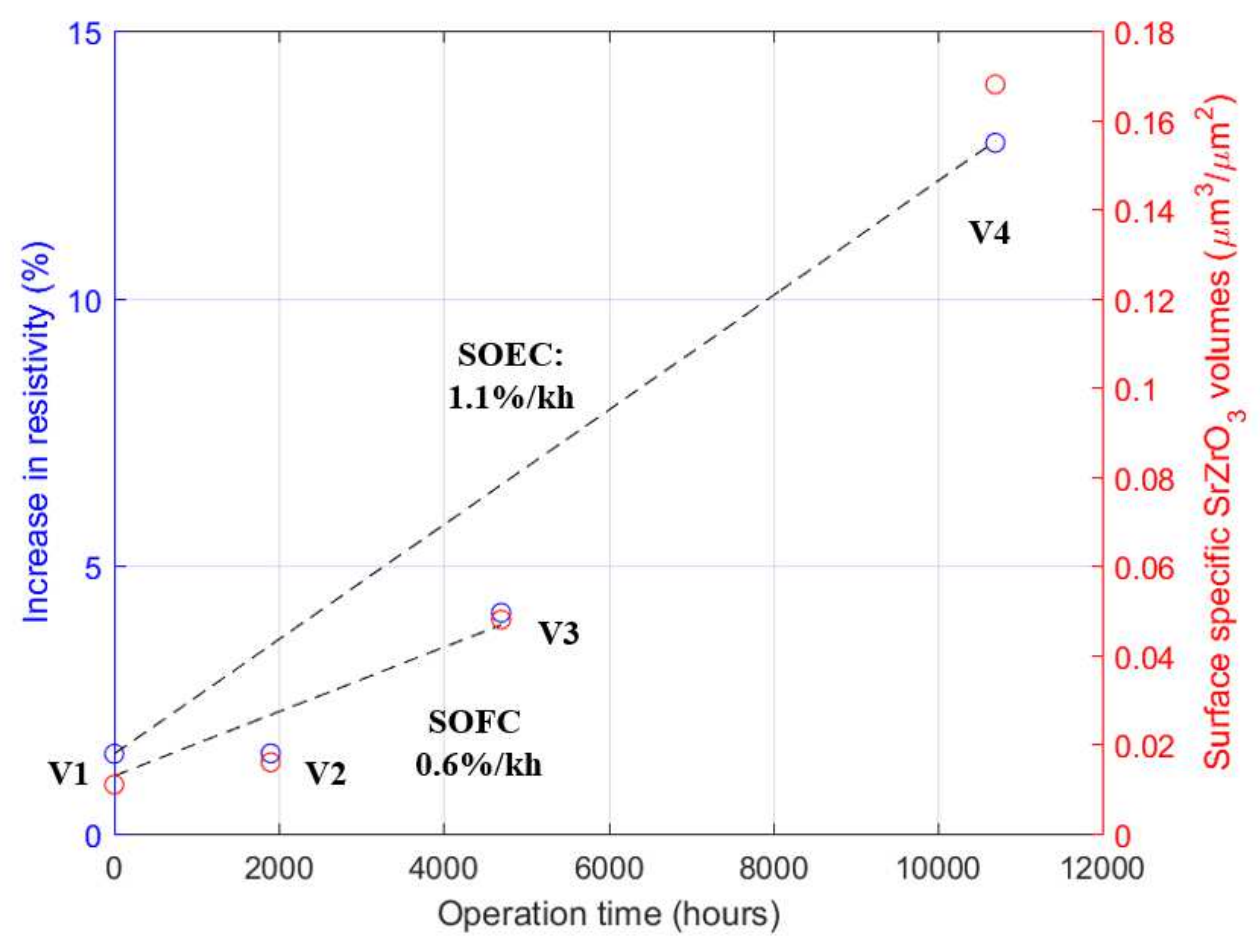

Figure 7. Time-lapse analysis of the increase in effective resistivity.

\section{Conclusion}

Differences in the morphology and topology of the $\mathrm{SrZrO}_{3}$ secondary phase detected in the interface region between the YZS electrolyte and GDC blocking layer were analyzed in cell samples in pristine state and after ageing in SOFC mode for $1900 \mathrm{~h}$ and $4700 \mathrm{~h}$, and after $10700 \mathrm{~h}$ of operation in SOEC conditions. The measurement of metric and topological properties indicates not only accumulation during operation, clearly predominant in SOEC conditions, but also changes in the microstructure. The size distribution of the secondary phase increases with operation time, resulting in the formation of a reticulated network after $10700 \mathrm{~h}$ in SOEC mode and the subset of the interfacial surface area in contact with the pore phase significantly decreases.

The detrimental effect on the effective conductivity of the interface, i.e. the cell performance, has been quantified by 3-D finite-element simulations. The effect of the $\mathrm{SrZrO}_{3}$ alone is limited in the pristine cell $(1.5 \%)$, but it increases by up to $13 \%$ after 10700h in SOEC mode.

The study does not clarify whether or not the strong accumulation in the V4 case is mainly ascribable to the extended operation duration or to the conditions in electrolysis mode. For this purpose, 3-D analysis including additional volumes of interest is required to comprehend the differences in microstructural alterations.

\section{Acknowledgments}

This work was supported by the Swiss EOS Holding contract 2014-0365 funding the $\mathrm{PhD}$ thesis of $\mathrm{G}$. Rinaldi. Part of the research leading to these results has received 
funding from the European Union's Seventh Framework Programme (FP7/2007-2013) Fuel Cells and Hydrogen Joint Undertaking (FCH-JU-2013-1) under grant agreements No 621207 (Project name: ENDURANCE) and No 621173 (Project name: SOPHIA).

\section{References}

1. L. Kindermann, D. Das, H. Nickel, K. Hilpert, Solid State Ionics, 89, 215 (1996).

2. F. Wang, M. Nishi, M. E. Brito, H. Kishimoto, K. Yamaji, H. Yokokawa, T, Horita, J. of Power Sources, 258, 281 (2014).

3. A. Tsoga, A. Gupta, A. Naoumidis, P. Nikolopoulos, Acta Materialia, 48, 4709 (2000).

4. R. Kiebach W. Zhang, W. Zhang, M. Chen, K. Norrman, H. Wang, J. R. Bowen, R. Barfod, P. Vang Hendriksen, J. of Power Sources, 283, 151 (2015).

5. F. Tietz, D. Sebold, A. Brisse, J. Schefold, J. of Power Sources, 223, 129 (2013).

6. D. The, S. Grieshammer, M. Schroeder, M. Martin, M. Al Daroukh, F. Tietz, J. Schefold, A. Brisse, J. of Power Sources, 275, 901 (2015).

7. Knibbe, M. L. Traulsen, A. Hauch, S. D. Ebbesen, M. Mogensen, J. of The Electrochemical Society, 157, B1209 (2010).

8. G. Rinaldi, S. Diethelm, E. Oveisi, P. Burdet, J. Van herle, D.Montinaro, Q. Fu, A. Brisse, Fuel Cells, (2017).

9. A. Nakajo, A. P. Cocco, M. B. DeGostin, A.A. Peracchio, B.N. Cassenti, M. Cantoni, J. Van herle, W. K. S. Chiu, J. of Power Sources 325, 786 (2016).

10. F. W. Poulsen, N. van der Puil, Solid State Ionics, 53, 777 (1992).

11. T. Kanit, S. Forest, I. Galliet, V. Mounoury, D.Jeulin, Int. J. of Solids and Structures, 40, 3647 (2003). 